\title{
A Study of Convolution Models for Background Correction of BeadArrays
}

\author{
Rohmatul Fajriyah \\ Graz University of Technology and Universitas Islam Indonesia
}

\begin{abstract}
The robust multi-array average (RMA), since its introduction in Irizarry, Bolstad, Collin, Cope, Hobbs, and Speed (2003a); Irizarry, Hobbs, Collin, Beazer-Barclay, Antonellis, Scherf, and Speed (2003b); Irizarry, Wu, and Jaffee (2006), has gained popularity among bioinformaticians. It has evolved from the exponential-normal convolution to the gamma-normal convolution, from single to two channels and from the Affymetrix to the Illumina platform.

The Illumina design provides two probe types: the regular and the control probes. This design is very suitable for studying the probability distribution of both and one can apply a convolution model to compute the true intensity estimator.

In this paper, we study the existing convolution models for background correction of Illumina BeadArrays in the literature and give a new estimator for the true intensity, assuming that the intensity value is exponentially or gamma distributed and the noise has lognormal distribution.

Our study shows that one of our proposed models, the gamma-lognormal with the method of moments for parameters estimation, is the optimal one for the benchmarking data set with benchmarking criteria, while the gamma-normal model has the best performance for the benchmarking data set with simulation criteria.

For the publicly available data sets, the gamma-normal and the exponential-gamma models with maximum likelihood estimation method can not be used and our proposed models exponential-lognormal and gamma-lognormal have the best performance, showing a moderate error in background correction and in the parametrization.
\end{abstract}

Keywords: convolution, background correction, BeadArrays.

\section{Introduction}

There are various processes in producing data from microarray experiments and each process contributes noise to the data. The noise can be of two types, biological and non-biological. Non-biological noise should be avoided or at least minimized.

Sources for the non-biological noise are, for example, the chip itself, the scanner, or fluctuations in the electric network. Therefore, the data needs to be adjusted. The pre-processing will adjust the intensity value (Huber, Irizarry, and Gentleman 2005a; Huber, von Heydebreck, and Vingron 2005b) and provides an estimate of the true intensity. 
To estimate the intensity value, researchers proposed additive and multiplicative models and also additive-muliplicative error models, see e.g. Huber, von Heydebreck, and Vingron (2004). In case of additive models, the underlying distribution is generally chosen as normal (log-normal), exponential, or a Gamma- $t$ mixture in the parametric approach (Allen, Chen, and Xie (2009), Bolstad, Irizarry, Astrand, and Speed (2003), Chen, Xie, and Story (2011), Hochreiter, Djork-Arné, and Obermayer (2006), Irizarry et al. (2003a,b, 2006), and Plancade, Rozenholc, and Lund (2011, 2012)).

Irizarry et al. (2003a,b, 2006) and Bolstad et al. (2003), on the Affymetrix platform, have estimated the true intensity values based on a convolution model in the background correction step of their robust multi-array average (RMA) pre-processing method. They assumed that the true intensity is exponentially distributed and the background noise is normally distributed.

Plancade et al. (2011, 2012) showed that the RMA model (in Bolstad et al. (2003) and Irizarry et al. (2003a,b, 2006)) does not fit Illumina BeadArrays: using the exponential-normal convolution leads to a large distance between the observed and the modeled intensities. They proposed, instead, the implementation of a gamma distribution for the intensity value and normal distribution for the noise.

The simulation study of Plancade et al. (2011, 2012) showed that the gamma-normal model performs better than the existing exponential-normal convolution model, giving a more accurate and correct fit for the observed intensities in Illumina BeadArray.

Using a Gamma distribution for the intensity values in Illumina BeadArrays has been first suggested by Xie, Wang, and Story (2009).

The studies of Baek, Son, and MacLachlan (2007) (on the background correction of the image processing) and Chen et al. (2011) show that the noise distribution is usually skewed in different degrees. In their studies, based on simulated and real data sets, Baek et al. (2007) conclude that the gamma distribution is well suited for the noise. It accounts for the intensities with a positive lower bound and is very flexible in its shape, including asymmetric exponential type and symmetric normal type.

The proposed convolution of exponential-gamma distribution by Chen et al. (2011) improves the intensity estimation and the detection of differentially expressed genes in the case when the intensity to noise ratio is large and the noise has a skewed distribution.

In view of the remarks above, it is natural to model both the true intensity and the background noise in Illumina BeadArrays as gamma distributed. In an earlier version of this paper we have developed an estimator for the true intensity based on the gamma-gamma convolution model of RMA. However, this model does not fit very well the Illumina benchmarking data set. Independently, Triche, Weisenberger, Berg, Laird, and Siegmund (2013) proposed and applied the gamma-gamma model to pre-process Illumina methylation arrays.

In this paper we introduce a new model for background correction in Illumina BeadArrays where the true intensity value is exponentially or gamma distributed and the noise has a lognormal distribution. As we will see, this model avoids the difficulties with the gammagamma model and has an overall satisfactory performance.

We note that a new method reducing the bias of the maximum likelihood estimator of the shape parameter of the gamma distribution was proposed by Zhang (2013). But since our samples are very large, bias is not a problem in our studies.

We compare the performance of the models on the Illumina spike-in data set, based on various criteria: root and mean square error (RMSE), $L_{1}$ error, Kullback-Leibler (K-L) coefficient, and some adapted criteria from Affycomp (Cope, Irizarry, Jaffee, Wu, and Speed 2004). These criteria are measuring the reproducibility, accuracy, precision, specificity, and sensitivity of the expression measure of each model. We then provide a simulation study to measure the consistency of the error of background correction and the parametrization. The description and some details on these criteria and simulation can be found at http://rfajriyah.staff. 
uii.ac.id/category/supplementary/

Our paper is organized as follows. In Section 2 we review previous work related to the background correction for Illumina BeadArrays. Our proposed models are described in Sections 3.1 and 3.2. Section 3.3 explains the benchmarking and the simulation studies on the benchmarking data set and Section 3.4 compares the performance of all models in the public data sets. Finally, Section 4 states the conclusions and indications of future work.

\section{Previous work}

Affymetrix is the pioneer and most widely used platform for microarray gene expression experiments. The tools and algorithms to handle the data are numerous, both free and commercial. Some methods for pre-processing are available. Examples for the background correction step are: MAS5.0 by Affymetrix, multiplicative model based expression index (MMBE) by Li and Wong (2001), RMA in Irizarry et al. (2003a,b, 2006) and Bolstad et al. (2003), GC-RMA by Wu, Irizarry, Gentleman, Martinez-Murillo, and Spencer (2004) and maximum likelihood estimation based on the normal-exponential convolution model by Silver, Ritchie, and Smyth (2009).

Illumina is one of the alternative platforms and is increasingly popular. A few statistical methods have been developed for BeadArray data and there is no consensus yet for the pre-processing steps (Shi, Oshlack, and Smyth 2010). Xie et al. (2009) mention that for the background correction step, Illumina bead studio gives two options (no background correction and background substraction) and the packages for BeadArrays in $\mathrm{R}$ provide three options (no background correction, background substraction and RMA background correction).

Ding, Xie, Park, Xiao, and Story (2008) extended the RMA model by proposing the modelbased background correction method (MBCB) and showed that their model leads to a more precise determination of the gene expression and a better biological interpretation of Illumina BeadArray data.

The studies of Chen et al. (2011) and Plancade et al. (2011, 2012) show that their background correction models are made by adapting the RMA Affymetrix model. As Forcheh, Verbeke, Kasim, Lin, Shkedy, Talloen, Gohlmann, and Clement (2012), pointed out, most preprocessing methods for Illumina BeadArrays are taken from the Affymetrix microarray platform.

In general, the background correction is applied toward each array, where in each array there are probes, probesets and genes (terminology for the Affymetrix platform) or bead and beadtype level probes (terminology for the Illumina platform).

At the Illumina platform, each gene is only targeted by one bead-type, which has been represented by about 30 time replications. If we can have a raw benchmarking data set, then it is possible to have all bead-type level probes of the raw data intensities.

The current publicly available benchmarking data set for the Illumina platform is the raw data from the bead studio, which is the average of the bead-type level probes, not background corrected and of unnormalized intensity. Therefore, the background correction in this paper is applied to the gene intensity in each array.

Suppose we have $J$ arrays and for each array there are $I$ regular genes. Our convolution model is as follows:

$$
P_{i j}=S_{i j}+B_{i j}, \quad i=1, \ldots, I, \quad j=1, \ldots, J,
$$

where $P_{i j}, S_{i j}$, and $B_{i j}$ are the observed signal, true signal, and noise intensity, respectively. The $S_{i j}$ are i.i.d. and so are the $B_{i j}$; the $S_{i j}$ are independent of the $B_{i j}$. The $P_{i j}$ are observable. Our task is to recover the unknown signals $S_{i j}$ from the $P_{i j}$. To do this, for each array $j$ we also have $M$ observable noise intensities $B_{0 m j}, m=1, \ldots, M$; the $B_{0 m j}$ are i.i.d. with the same distribution as the $B_{i j}$ and are independent of all of the $S_{i j}, B_{i j}$. The $S_{i j}$ and $B_{i j}$ have a known type of distribution (exponential, gamma, normal, lognormal) with unknown parameters. 


\subsection{Background correction by RMA}

The RMA method was developed for the Affymetrix platform, where the design of arrays is different from the Illumina one. In this platform, one has perfect match and mismatch probe design. The RMA uses only the perfect match probe, which is the targeted probe of the intended gene. For those not familiar with the Affymetrix platform, refer to Bolstad (2004), Bolstad et al. (2003), and Irizarry et al. (2003a,b, 2006) for further information.

In modeling the intensity values, the RMA model (Bolstad et al. (2003) and Irizarry et al. (2003a,b, 2006)) assumes that the intensity values are affected by the noise of the chip. By referring to Equation (1), in the RMA model $P_{i j}$ is the observed bead-type level probe intensity, $S_{i j}$ is the true signal with $S_{i j} \sim f_{1}\left(s_{i j} ; \theta_{j}\right)=\operatorname{Exp}\left(\theta_{j}\right), \theta_{j}>0$, and $B_{i j}$ is the background noise of the chip with $B_{i j} \sim f_{2}\left(b_{i j} ; \mu_{j}, \sigma_{j}^{2}\right)=\mathcal{N}\left(\mu_{j}, \sigma_{j}^{2}\right), \mu_{j} \in \mathbb{R}, \sigma_{j}^{2}>0$. To avoid negative intensity values, we truncate $B_{i j}$ at 0 from below, i.e. we replace $B_{i j}$ by $\max \left\{B_{i j}, 0\right\}$; this will not change its density function $f_{2}\left(b_{i j} ; \mu_{j}, \sigma_{j}^{2}\right)$ for $b_{i j}>0$.

Assuming independence, the joint density of the two-dimensional random variable $\left(S_{i j}, B_{i j}\right)$ is

$$
f_{S_{i j}, B_{i j}}\left(s_{i j}, b_{i j} ; \mu_{j}, \sigma_{j}^{2}, \theta_{j}\right)=\theta_{j} \exp \left\{-\theta_{j} s_{i j}\right\} f_{2}\left(b_{i j} ; \mu_{j}, \sigma_{j}^{2}\right), \quad b_{i j}, s_{i j}>0 .
$$

Furthermore, the transformation formula for two-dimensional densities gives that joint density of $S$ and $P$ is

$$
\begin{aligned}
f_{S_{i j}, P_{i j}}\left(s_{i j}, p_{i j} ; \mu_{j}, \sigma_{j}^{2}, \theta_{j}\right) \\
\quad=\theta_{j} \exp \left\{\frac{\theta_{j}^{2} \sigma_{j}^{2}}{2}-\theta_{j}\left(p_{i j}-\mu_{j}\right)\right\} f_{2}\left(s_{i j} ; \mu_{S . P, j}, \sigma_{j}^{2}\right), \quad 0<s_{i j}<p_{i j},
\end{aligned}
$$

where $\mu_{S . P, j}=p_{i j}-\mu_{j}-\theta_{j} \sigma_{j}^{2}$. From (2) we get the marginal density of $P_{i j}$ and the conditional density of $S_{i j}$ given $P_{i j}$ as

$$
\begin{gathered}
f_{P_{i j}}\left(p_{i j} ; \theta_{j}, \mu_{j}, \sigma_{j}^{2}\right)=\theta_{j} \exp \left\{\frac{\theta_{j}^{2} \sigma_{j}^{2}}{2}-\theta_{j}\left(p_{i j}-\mu_{j}\right)\right\}\left(\Phi\left(\frac{\mu_{S . P, j}}{\sigma_{j}}\right)+\Phi\left(\frac{p_{i j}-\mu_{S . P, j}}{\sigma_{j}}\right)-1\right) \\
f_{S_{i j} \mid P_{i j}}\left(s_{i j} \mid p_{i j} ; \mu_{S . P, j}, \sigma_{j}^{2}\right)=\frac{f_{2}\left(s_{i j} ; \mu_{S . P, j}, \sigma_{j}^{2}\right)}{\Phi\left(\frac{\mu_{S . P, j}}{\sigma_{j}}\right)+\Phi\left(\frac{p_{i j}-\mu_{S . P, j}}{\sigma_{j}}\right)-1} .
\end{gathered}
$$

The background adjusted intensity is computed as the conditional expectation of the true signal given the observed intensity, i.e.

$$
\mathrm{E}\left(S_{i j} \mid P_{i j}=p_{i j}\right)=\frac{1}{\Phi\left(\frac{\mu_{S . P, j}}{\sigma_{j}}\right)+\Phi\left(\frac{p_{i j}-\mu_{S . P, j}}{\sigma_{j}}\right)-1} \int_{0}^{p_{i j}} s_{i j} f_{2}\left(s_{i j} ; \mu_{S . P, j}, \sigma_{j}^{2}\right) d s_{i j} .
$$

The substitution $s_{i j}=\mu_{S . P, j}+\sigma_{j} t$ yields

$$
\begin{aligned}
& \int_{0}^{p_{i j}} s_{i j} f_{2}\left(s_{i j} ; \mu_{S . P, j}, \sigma_{j}^{2}\right) d s_{i j} \\
& \quad=\mu_{S . P, j}\left(\Phi\left(\frac{p_{i j}-\mu_{S . P, j}}{\sigma_{j}}\right)+\Phi\left(\frac{\mu_{S . P, j}}{\sigma_{j}}\right)-1\right)+\sigma_{j}\left(\phi\left(\frac{\mu_{S . P, j}}{\sigma_{j}}\right)-\phi\left(\frac{p_{i j}-\mu_{S . P, j}}{\sigma_{j}}\right)\right)
\end{aligned}
$$

and thus (see Bolstad et al. (2003))

$$
\mathrm{E}\left(S_{i j} \mid P_{i j}=p_{i j}\right)=\mu_{S . P, j}+\sigma_{j} \frac{\phi\left(\frac{\mu_{S . P, j}}{\sigma_{j}}\right)-\phi\left(\frac{p_{i j}-\mu_{S . P, j}}{\sigma_{j}}\right)}{\Phi\left(\frac{\mu_{S . P, j}}{\sigma_{j}}\right)+\Phi\left(\frac{p-\mu_{S . P, j}}{\sigma_{j}}\right)-1} .
$$

Note that modelling the noise as a truncated normal variable has the consequence that the noise equals 0 with a positive probability $p_{0}$, a rather unpleasant feature of the model. As 
pointed out in Xie et al. (2009), however, in practical cases $p_{0}$ is rather small, so this problem can be disregarded. To avoid this difficulty, one can model the noise as the absolute value of a $\mathcal{N}\left(\mu, \sigma^{2}\right)$ variable, which changes the calculations above. However, since in this paper we will provide a background correction model fitting the reality considerably better, we do not give the details here.

\subsection{Exponential-normal MBCB}

Xie et al. (2009) use the same underlying distributions in (1) for background correction. The difference with the RMA (Bolstad et al. (2003) and Irizarry et al. (2003a,b, 2006)) are

1. Xie et al. (2009) use $+\infty$ as the upper bound of the integral to compute the marginal density function and the conditional expectation of the true intensity value. On the other hand, RMA uses $p$ as the upper bound of the integration.

The background corrected intensity value of Xie et al. (2009) is

$$
\mathrm{E}\left(S_{i j} \mid P_{i j}=p_{i j}\right)=\mu_{S . P, j}+\sigma_{j} \frac{\phi\left(\frac{\mu_{S . P, j}}{\sigma_{j}}\right)}{\Phi\left(\frac{\mu_{S . P, j}}{\sigma_{j}}\right)} .
$$

2. Under the convolution model (1), where the true intensity value is assumed exponentially distributed and the noise is normally distributed, we need to estimate the parameters $\theta_{j}$, $\mu_{j}$, and $\sigma_{j}^{2}$. Xie et al. (2009) offer three estimation methods: the method of moments, maximum likelihood estimation, and a Bayesian approach. On the other hand, RMA applies the ad-hoc method.

Ding et al. (2008) use the exponential-normal convolution model to correct the background of the Illumina platform by using Markov chain Monte Carlo simulation.

\subsection{Gamma-normal convolution}

Plancade et al. $(2011,2012)$ introduced gamma-normal convolution to model the background correction of Illumina BeadArray. The model is based on the RMA background correction of Affymetrix GeneChip. Plancade et al. (2011, 2012) assume that the intensity value is gamma distributed and the noise is normally distributed.

Under model (1), $f_{P_{i j}}$ is the convolution of $f_{S_{i j}}$ and $f_{B_{i j}}$. The background corrected intensity is computed as the conditional expectation of $S_{i j}$ given $P_{i j}=p_{i j}$, i.e.

$$
\mathrm{E}\left(S_{i j} \mid P_{i j}=p_{i j}\right)=\frac{\int s_{i j} f_{\alpha_{j}, \beta_{j}}^{\text {gam }}\left(s_{i j}\right) f_{\mu_{j}, \sigma_{j}}^{\text {norm }}\left(p_{i j}-s_{i j}\right) d s_{i j}}{\int f_{\alpha_{j}, \beta_{j}}^{\text {gam }}\left(s_{i j}\right) f_{\mu_{j}, \sigma_{j}}^{\text {norm }}\left(p_{i j}-s_{i j}\right) d s_{i j}},
$$

where

$$
f_{\alpha_{j}, \beta_{j}}^{\mathrm{gaam}}(s)=\frac{\beta_{j}^{\alpha_{j}} s^{\alpha_{j}-1} \exp \left(-\beta_{j} s\right)}{\Gamma\left(\alpha_{j}\right)}, \quad \alpha_{j}, \beta_{j}, s>0
$$

is the gamma density. When $S_{i j}$ is gamma distributed and $B_{i j}$ is normally distributed, then (4) has no analytic expression like (3). Plancade et al. (2011, 2012) implemented the Fast Fourier Transform to estimate the parameters and to correct the background. For the background correction with Fast Fourier Transform, the corrected intensity (4) is rewritten as

$$
\mathrm{E}\left(S_{i j} \mid P_{i j}=p_{i j}\right)=\frac{\alpha_{j} \beta_{j} \int f_{\alpha_{j}+1, \beta_{j}}^{\mathrm{gam}}\left(s_{i j}\right) f_{\mu_{j}, \sigma_{j}}^{\text {norm }}\left(p_{i j}-s_{i j}\right) d s_{i j}}{\int f_{\alpha_{j}, \beta_{j}}^{\mathrm{gam}}\left(s_{i j}\right) f_{\mu_{j}, \sigma_{j}}^{\text {norm }}\left(p_{i j}-s_{i j}\right) d s_{i j}},
$$

since $s_{i j} f_{\alpha_{j}, \beta_{j}}^{\text {gam }}\left(s_{i j}\right)=\alpha_{j} \beta_{j} f_{\alpha_{j}+1, \beta_{j}}^{\text {gam }}\left(s_{i j}\right)$ is valid for every $s_{i j}>0$. 


\subsection{Exponential-gamma convolution}

Under model (1), Chen et al. (2011) proposed for the distribution of the true intensity and its noise the exponential and gamma distribution, respectively. Therefore, $S_{i j} \sim f_{1}\left(s_{i j} ; \theta_{j}\right)=$ $\operatorname{Exp}\left(\theta_{j}\right)$ and $B_{i j} \sim f_{2}\left(b_{i j} ; \alpha_{j}, \beta_{j}\right)=\operatorname{Gamma}\left(\alpha_{j}, \beta_{j}\right)$, where $s_{i j}, b_{i j}, \theta_{j}, \alpha_{j}, \beta_{j}>0$.

The corrected background intensity of Chen et al. (2011) is

$$
\mathrm{E}\left(S_{i j} \mid P_{i j}=p_{i j}\right)=p_{i j}-\frac{\int_{0}^{p_{i j}} b_{i j}^{\alpha_{j}} \exp \left(-\left(\beta_{j}-\theta_{j}\right) b_{i j}\right) d b_{i j}}{\int_{0}^{p_{i j}} b_{i j}^{\alpha_{j}-1} \exp \left(-\left(\beta_{j}-\theta_{j}\right) b_{i j}\right) d b_{i j}} .
$$

\section{Results}

Now we present the results of the two proposed convolution models: the exponential-lognormal convolution in Section 3.1 and the gamma-lognormal convolution in Section 3.2. In each section, the formula for the background corrected intensity value is derived and methods to estimate the parameters are explained. Section 3.3 present the benchmarking results, i.e. a performance comparison of all models at the Illumina spike-in data set. Section 3.4 present the performance comparison of all models for the public data sets. The simulation study results are presented in Sections 3.3 and 3.4.

For further details on the benchmarking criteria, supplemental plots and simulations see Supplementary_Materials at http://rfajriyah.staff.uii.ac.id/category/supplementary/.

\subsection{Exponential-lognormal convolution}

Background correction Consider model (1) when the true intensity $S_{i j}$ is exponentially distributed, i.e.

$$
S_{i j} \sim f_{1}\left(s_{i j} ; \theta_{j}\right)=\theta_{j} \exp \left(-\theta_{j} s_{i j}\right), \quad \theta_{j}, s_{i j}>0
$$

and the background noise $B_{i j}$ is lognormally distributed, i.e.

$$
B_{i j} \sim f_{2}\left(b_{i j} ; \mu_{j}, \sigma_{j}^{2}\right)=\frac{1}{b_{i j} \sigma_{j} \sqrt{2 \pi}} \exp \left(-\frac{\left(\log b_{i j}-\mu_{j}\right)^{2}}{2 \sigma_{j}^{2}}\right), \quad \mu_{j} \in \mathbb{R}, \sigma_{j}^{2}, b_{i j}>0 .
$$

Then the joint density function of $S_{i j}$ and $B_{i j}$ equals

$$
f_{S_{i j}, B_{i j}}\left(s_{i j}, b_{i j}\right)=\frac{\theta_{j} \exp \left(-\theta_{j} s_{i j}\right)}{b_{i j} \sigma_{j} \sqrt{2 \pi}} \exp \left(-\frac{\left(\log b_{i j}-\mu_{j}\right)^{2}}{2 \sigma_{j}^{2}}\right),
$$

and thus the joint density function of $S_{i j}$ and $P_{i j}$ is

$$
f_{S_{i j}, P_{i j}}\left(s_{i j}, p_{i j}\right)=\frac{\theta_{j} \exp \left(-\theta_{j} s_{i j}\right)}{\left(p_{i j}-s_{i j}\right) \sigma_{j} \sqrt{2 \pi}} \exp \left(-\frac{\left(\log \left(p_{i j}-s_{i j}\right)-\mu_{j}\right)^{2}}{2 \sigma_{j}^{2}}\right), \quad s_{i j}<p_{i j} .
$$

Consequently, the marginal density function of $P_{i j}$ equals

$$
f_{P_{i j}}\left(p_{i j}\right)=\int_{0}^{p_{i j}} \frac{\theta_{j} \exp \left(-\theta_{j} s_{i j}\right)}{\left(p_{i j}-s_{i j}\right) \sigma_{j} \sqrt{2 \pi}} \exp \left(-\frac{\left(\log \left(p_{i j}-s_{i j}\right)-\mu_{j}\right)^{2}}{2 \sigma_{j}^{2}}\right) d s_{i j} .
$$


Using the substitution $\log \left(p_{i j}-s_{i j}\right)=z_{i j}$ we get

$$
\begin{aligned}
f_{P_{i j}}\left(p_{i j}\right) & =\int_{-\infty}^{\log p_{i j}} \frac{\theta_{j} \exp \left(-\theta_{j}\left(p_{i j}-e^{z_{i j}}\right)\right)}{\sigma_{j} \sqrt{2 \pi}} \exp \left(-\frac{\left(z_{i j}-\mu_{j}\right)^{2}}{2 \sigma_{j}^{2}}\right) d z_{i j} \\
& =\frac{\theta_{j} \exp \left(-\theta_{j} p_{i j}\right)}{\sigma_{j} \sqrt{2 \pi}} \int_{-\infty}^{\log p_{i j}} \exp \left(-\frac{\left(z_{i j}-\mu_{j}\right)^{2}}{2 \sigma_{j}^{2}}\right) \sum_{k=0}^{\infty} \frac{\theta_{j}^{k} e^{k z_{i j}}}{k !} d z_{i j} \\
& =\frac{\theta_{j} \exp \left(-\theta_{j} p_{i j}\right)}{\sigma_{j} \sqrt{2 \pi}} \sum_{k=0}^{\infty} \frac{\theta_{j}^{k}}{k !} \int_{-\infty}^{\log p_{i j}} \exp \left(-\frac{\left(z_{i j}-\mu_{j}\right)^{2}}{2 \sigma_{j}^{2}}+k z_{i j}\right) d z_{i j} \\
& =\frac{\theta_{j} \exp \left(-\theta_{j} p_{i j}\right)}{\sigma_{j} \sqrt{2 \pi}} \sum_{k=0}^{\infty} \frac{\theta_{j}^{k}}{k !} \exp \left(k\left(\mu_{j}+\frac{k}{2} \sigma_{j}^{2}\right)\right) \int_{-\infty}^{\log p_{i j}} \exp \left(-\frac{\left(z_{i j}-\left(\mu_{j}+k \sigma_{j}^{2}\right)\right)^{2}}{2 \sigma_{j}^{2}}\right) d z_{i j} \\
& =\theta_{j} \exp \left(-\theta_{j} p_{i j}\right) C_{a, j},
\end{aligned}
$$

where

$$
C_{a, j}=\sum_{k=0}^{\infty} \frac{\theta_{j}^{k}}{k !} \exp \left(k\left(\mu_{j}+\frac{k}{2} \sigma_{j}^{2}\right)\right) \Phi\left(\frac{\log p_{i j}-\left(\mu_{j}+k \sigma_{j}^{2}\right)}{\sigma_{j}}\right) .
$$

The conditional density function of $S_{i j}$ given $P_{i j}=p_{i j}$ is now obtained as

$$
f_{S_{i j} \mid P_{i j}}\left(s_{i j} \mid p_{i j}\right)=\frac{\exp \left(\theta_{j}\left(p_{i j}-s_{i j}\right)\right)}{\left(p_{i j}-s_{i j}\right) \sigma_{j} \sqrt{2 \pi} C_{a, j}} \exp \left(-\frac{\left(\log \left(p_{i j}-s_{i j}\right)-\mu_{j}\right)^{2}}{2 \sigma_{j}^{2}}\right)
$$

with conditional mean

$$
\mathrm{E}\left(S_{i j} \mid P_{i j}=p_{i j}\right)=\frac{\exp \left(\theta_{j} p_{i j}\right)}{C_{a, j}} \int_{0}^{p_{i j}} \frac{s_{i j} \exp \left(-\theta_{j} s_{i j}\right)}{\left(p_{i j}-s_{i j}\right) \sigma_{j} \sqrt{2 \pi}} \exp \left(-\frac{\left(\log \left(p_{i j}-s_{i j}\right)-\mu_{j}\right)^{2}}{2 \sigma_{j}^{2}}\right) d s_{i j} .
$$

Using the substitution $\log \left(p_{i j}-s_{i j}\right)=z_{i j}$, this equals

$$
\begin{aligned}
\mathrm{E}\left(S_{i j} \mid P_{i j}=p_{i j}\right) & =\frac{p_{i j}}{C_{a, j}} \int_{-\infty}^{\log p_{i j}} \frac{\left(1-\frac{e^{z_{i j}}}{p_{i j}}\right) \exp \left(\theta_{j} e^{z_{i j}}\right)}{\sigma_{j} \sqrt{2 \pi}} \exp \left(-\frac{\left(z_{i j}-\mu_{j}\right)^{2}}{2 \sigma_{j}^{2}}\right) d z_{i j} \\
& =\frac{p_{i j}}{C_{a, j}}\left[C_{a, j}-\int_{-\infty}^{\log p_{i j} \frac{e^{z_{i j}}}{p_{i j}} \exp \left(\theta_{j} e^{z_{i j}}\right)}\right. \\
\sigma_{j} \sqrt{2 \pi} & \left.\exp \left(-\frac{\left(z_{i j}-\mu_{j}\right)^{2}}{2 \sigma_{j}^{2}}\right) d z_{i j}\right] \\
& =p_{i j}-\frac{\exp \left(\mu_{j}+\frac{\sigma_{j}^{2}}{2}\right)}{C_{a, j}} \int_{-\infty}^{\log p_{i j}} \frac{\exp \left(\theta_{j} e^{z_{i j}}\right)}{\sigma_{j} \sqrt{2 \pi}} \exp \left(-\frac{\left(z_{i j}-\left(\mu_{j}+\sigma_{j}^{2}\right)\right)^{2}}{2 \sigma_{j}^{2}}\right) d z_{i j} \\
& =p_{i j}-\frac{\exp \left(\mu_{j}+\frac{\sigma_{j}^{2}}{2}\right)}{C_{a, j}} \sum_{k=0}^{\infty} \frac{\theta_{j}^{k}}{k !} \exp \left(k\left(\mu_{j}+\frac{k+2}{2} \sigma_{j}^{2}\right)\right) \\
& \times \int_{-\infty}^{\log p_{i j}} \frac{1}{\sigma_{j} \sqrt{2 \pi}} \exp \left(-\frac{\left(z_{i j}-\left(\mu_{j}+(k+1) \sigma_{j}^{2}\right)\right)^{2}}{2 \sigma_{j}^{2}}\right) d z_{i j} \\
& =p_{i j}-\frac{C_{b, j}}{C_{a, j}} \exp \left(\mu_{j}+\frac{\sigma_{j}^{2}}{2}\right)
\end{aligned}
$$

where

$$
C_{b, j}=\sum_{k=0}^{\infty} \frac{\theta_{j}^{k}}{k !} \exp \left(k\left(\mu_{j}+\frac{k+2}{2} \sigma_{j}^{2}\right)\right) \Phi\left(\frac{\log p_{i j}-\left(\mu_{j}+(k+1) \sigma_{j}^{2}\right)}{\sigma_{j}}\right) .
$$


Parameter estimation To estimate the parameters $\theta_{j}, \mu_{j}$, and $\sigma_{j}^{2}, j=1, \ldots, J$ in the exponential-lognormal model we can use various methods.

1. Maximum likelihood estimation (MLE):

This is implemented by applying the optim function in $\mathrm{R}$ to maximize the log-likelihood function of the $j$ th array

$$
\sum_{i=1}^{I}\left(\log \theta_{j}-\theta_{j} p_{i j}+\log C_{a, j ; K}\right)+\sum_{m=1}^{M}\left(-\frac{\left(\log b_{0 m j}-\mu_{j}\right)^{2}}{2 \sigma_{j}^{2}}-\frac{1}{2} \log \left(2 \pi \sigma_{j}^{2} b_{0 m j}^{2}\right)\right),
$$

where $p_{i j}$ and $b_{0 m j}$ are the observed values of $P_{i j}$ and $B_{0 m j}$. Note that $C_{a, j}$ in the log-likelihood function is defined by the infinite series at the end of the previous section. However, the terms of this infinite series decrease very rapidly and thus we can cut off the series at a proper index $K$ giving $C_{a, j ; K}$, making it suitable for its computation in R. The index $K$ is chosen as the smallest integer for which $\left|\left(C_{a, j ; K}-C_{a, j ; K-1}\right) / C_{a, j ; K-1}\right|<0.001$ holds.

2. Method of moments:

Note that the method of moments estimator of the parameter $\theta$ in an exponential distribution is the reciprocal of the sample mean. Since the $S_{i j}$ are not observable, but the $B_{0 m j}$ are, we consider Equation (1) and estimate $1 / \theta_{j}$ by the difference mean $\left(p_{i j}\right)-$ mean $\left(b_{0 m j}\right)$. Further, the parameters $\mu_{j}$ and $\sigma_{j}^{2}$ in the lognormal part are estimated by the sample mean and variance of the observed $\log b_{0 m j}$ values.

3. Plug-in estimator:

(a) We calculate the MLE of the parameter in the model of $S_{i j}$ by utilizing the observed sample $p_{i j}$ instead of $s_{i j}$. This is justified by the fact that, in some sense, the distribution of $S_{i j}$ is similar to the distribution of $P_{i j}$.

(b) We estimate $\mu_{j}$ and $\sigma_{j}^{2}$ through MLE based on $B_{0 m j}$ as described above.

\subsection{Gamma-lognormal convolution}

Background correction Consider now model (1) and assume that the true intensity $S_{i j}$ is gamma distributed, i.e.

$$
S_{i j} \sim f_{1}\left(s_{i j} ; \alpha_{j}, \beta_{j}\right)=\frac{\beta_{j}^{\alpha_{j}} s_{i j}^{\alpha_{j}-1} \exp \left(-s_{i j} \beta_{j}\right)}{\Gamma\left(\alpha_{j}\right)}, \quad \alpha_{j}, \beta_{j}, s_{i j}>0,
$$

and that the background noise $B_{i j}$ is lognormally distributed, i.e.

$$
B_{i j} \sim f_{2}\left(b_{i j} ; \mu_{j}, \sigma_{j}^{2}\right)=\frac{1}{b_{i j} \sigma_{j} \sqrt{2 \pi}} \exp \left(-\frac{\left(\log b_{i j}-\mu_{j}\right)^{2}}{2 \sigma_{j}^{2}}\right), \quad \mu_{j} \in \mathbb{R}, \sigma_{j}^{2}>0 .
$$

The joint density function of $S_{i j}$ and $B_{i j}$ is

$$
f_{S_{i j}, B_{i j}}\left(s_{i j}, b_{i j}\right)=\frac{\beta_{j}^{\alpha_{j}} s_{i j}^{\alpha_{j}-1} \exp \left(-s_{i j} \beta_{j}\right)}{\Gamma\left(\alpha_{j}\right)} \frac{1}{b_{i j} \sigma_{j} \sqrt{2 \pi}} \exp \left(-\frac{\left(\log b_{i j}-\mu_{j}\right)^{2}}{2 \sigma_{j}^{2}}\right)
$$

and thus the joint density function of $S_{i j}$ and $P_{i j}$ is

$$
f_{S_{i j}, P_{i j}}\left(s_{i j}, p_{i j}\right)=\frac{\beta_{j}^{\alpha_{j}} s_{i j}^{\alpha_{j}-1} \exp \left(-s_{i j} \beta_{j}\right)}{\Gamma\left(\alpha_{j}\right)} \frac{1}{\left(p_{i j}-s_{i j}\right) \sigma_{j} \sqrt{2 \pi}} \exp \left(-\frac{\left(\log \left(p_{i j}-s_{i j}\right)-\mu_{j}\right)^{2}}{2 \sigma_{j}^{2}}\right) .
$$


Hence, the marginal density function of $P_{i j}$ is obtained as

$$
f_{P_{i j}}\left(p_{i j}\right)=\int_{0}^{p_{i j}} \frac{\beta_{j}^{\alpha_{j}} s_{i j}^{\alpha_{j}-1} \exp \left(-s_{i j} \beta_{j}\right)}{\Gamma\left(\alpha_{j}\right)} \frac{1}{\left(p_{i j}-s_{i j}\right) \sigma_{j} \sqrt{2 \pi}} \exp \left(-\frac{\left(\log \left(p_{i j}-s_{i j}\right)-\mu_{j}\right)^{2}}{2 \sigma_{j}^{2}}\right) d s_{i j} .
$$

Using the substitution $\log \left(p_{i j}-s_{i j}\right)=z_{i j}$ we get

$$
\begin{aligned}
f_{P_{i j}}\left(p_{i j}\right) & =\int_{-\infty}^{\log p_{i j}} \frac{\beta_{j}^{\alpha_{j}} p_{i j}^{\alpha_{j}-1}\left(1-\frac{e^{z_{i j}}}{p_{i j}}\right)^{\alpha_{j}-1} \exp \left(-p_{i j} \beta_{j}+e^{z_{i j} \beta_{j}}\right)}{\Gamma\left(\alpha_{j}\right) \sigma_{j} \sqrt{2 \pi}} \exp \left(-\frac{\left(z_{i j}-\mu_{j}\right)^{2}}{2 \sigma_{j}^{2}}\right) d z_{i j} \\
& =\frac{\beta_{j}^{\alpha_{j}} p_{i j}^{\alpha_{j}-1} \exp \left(-p_{i j} \beta_{j}\right)}{\Gamma\left(\alpha_{j}\right) \sigma_{j} \sqrt{2 \pi}} \int_{-\infty}^{\log p_{i j}}\left(1-\frac{e^{z_{i j}}}{p_{i j}}\right)^{\alpha_{j}-1} \exp \left(e^{z_{i j} \beta_{j}}\right) \exp \left(-\frac{\left(z_{i j}-\mu_{j}\right)^{2}}{2 \sigma_{j}^{2}}\right) d z_{i j} \\
& =\frac{\beta_{j}^{\alpha_{j}} p_{i j}^{\alpha_{j}-1} \exp \left(-p_{i j} \beta_{j}\right)}{\Gamma\left(\alpha_{j}\right)} C_{c, j},
\end{aligned}
$$

where

$C_{c, j}=\sum_{k=0}^{\infty} \sum_{n=0}^{\infty} \frac{(-1)^{k}\left(\begin{array}{c}\alpha_{j}-1 \\ k\end{array}\right)}{p_{i j}^{k} n !} \beta_{j}^{n} \exp \left((k+n)\left(\mu_{j}+(k+n) \frac{\sigma_{j}^{2}}{2}\right)\right) \Phi\left(\frac{\log p_{i j}-\left(\mu_{j}+(k+n) \sigma_{j}^{2}\right)}{\sigma_{j}}\right)$.

The conditional density function is now obtained as

$$
f_{S_{i j} \mid P_{i j}}\left(s_{i j} \mid p_{i j}\right)=\frac{\exp \left(\left(p_{i j}-s_{i j}\right) \beta_{j}\right) s_{i j}^{\alpha_{j}-1}}{C_{c, j} p_{i j}^{\alpha_{j}-1}\left(p_{i j}-s_{i j}\right) \sigma_{j} \sqrt{2 \pi}} \exp \left(-\frac{\left(\log \left(p_{i j}-s_{i j}\right)-\mu_{j}\right)^{2}}{2 \sigma_{j}^{2}}\right)
$$

with respective conditional mean

$$
\mathrm{E}\left(S_{i j} \mid P_{i j}=p_{i j}\right)=\frac{e^{p_{i j} \beta_{j}}}{C_{c, j} p_{i j}^{\alpha_{j}-1}} \int_{0}^{p_{i j}} \frac{s_{i j}^{\alpha_{j}} e^{-s_{i j} \beta_{j}}}{\left(p_{i j}-s_{i j}\right) \sigma_{j} \sqrt{2 \pi}} \exp \left(-\frac{\left(\log \left(p_{i j}-s_{i j}\right)-\mu_{j}\right)^{2}}{2 \sigma_{j}^{2}}\right) d s_{i j} .
$$

Substituting $\log \left(p_{i j}-s_{i j}\right)=z_{i j}$ this becomes

$$
\begin{aligned}
\mathrm{E}\left(S_{i j} \mid P_{i j}=p_{i j}\right) & =\frac{p_{i j}}{C_{c, j}} \int_{-\infty}^{\log p_{i j}} \frac{\left(1-\frac{e^{z_{i j}}}{p_{i j}}\right)^{\alpha_{j}} \exp \left(e^{z_{i j} \beta_{j}}\right)}{\sigma_{j} \sqrt{2 \pi}} \exp \left(-\frac{\left(z_{i j}-\mu_{j}\right)^{2}}{2 \sigma_{j}^{2}}\right) d z_{i j} \\
& =\frac{p_{i j} C_{d, j}}{C_{c, j}}
\end{aligned}
$$

where

$C_{d, j}=\sum_{k=0}^{\infty} \sum_{n=0}^{\infty} \frac{(-1)^{k}\left(\begin{array}{c}\alpha_{j} \\ k\end{array}\right)}{p_{i j}^{k} n !} \beta_{j}^{n} \exp \left((k+n)\left(\mu_{j}+(k+n) \frac{\sigma_{j}^{2}}{2}\right)\right) \Phi\left(\frac{\log p_{i j}-\left(\mu_{j}+(k+n) \sigma_{j}^{2}\right)}{\sigma_{j}}\right)$.

Parameter estimation To estimate the parameters $\alpha_{j}, \beta_{j}, \mu_{j}$, and $\sigma_{j}^{2}$ in (5), we can use either of the following methods.

1. Maximum likelihood estimation:

This is implemented by applying the optim function in R to maximize the log-likelihood function of the $j$ th array

$$
\begin{aligned}
& \sum_{i=1}^{I}\left(\log \left(C_{c, j ; K}\right)+\left(\alpha_{j}-1\right) \log \left(p_{i j}\right)-p_{i j} \beta_{j}+\alpha_{j} \log \left(\beta_{j}\right)-\log \left(\Gamma\left(\alpha_{j}\right)\right)\right) \\
& +\sum_{m=1}^{M}\left(-\frac{\left(\log b_{0 m j}-\mu_{j}\right)^{2}}{2 \sigma_{j}^{2}}-\frac{1}{2} \log \left(2 \pi \sigma_{j}^{2} b_{0 m j}^{2}\right)\right) .
\end{aligned}
$$

Similarly to the exponential-lognormal model, in the computation of $C_{c, j}$ the cutoff index $K$ is chosen according to the criteria $\left|\left(C_{c, j ; K}-C_{c, j ; K-1}\right) / C_{c, j ; K-1}\right|<0.002$. 
2. Method of moments:

The implementation of this method for the $j$ th array is done by recalling that in case of a gamma distribution with parameters $\alpha_{j}$ and $\beta_{j}$, the method of moments estimator for $\alpha_{j} / \beta_{j}$ and $\alpha_{j} / \beta_{j}^{2}$ is the sample mean and the sample variance, respectively. Thus, considering Equation (1) we get

$$
\hat{\beta}_{j}=\frac{\operatorname{mean}\left(s_{i j}\right)}{\operatorname{var}\left(s_{i j}\right)}=\frac{\operatorname{mean}\left(p_{i j}\right)-\operatorname{mean}\left(b_{0 m j}\right)}{\operatorname{var}\left(p_{i j}\right)-\operatorname{var}\left(b_{0 m j}\right)},
$$

as also

$$
\hat{\alpha}_{j}=\hat{\beta}_{j} \operatorname{mean}\left(s_{i j}\right)=\frac{\left(\operatorname{mean}\left(s_{i j}\right)\right)^{2}}{\operatorname{var}\left(s_{i j}\right)}=\frac{\left(\operatorname{mean}\left(p_{i j}\right)-\operatorname{mean}\left(b_{0 m j}\right)\right)^{2}}{\operatorname{var}\left(p_{i j}\right)-\operatorname{var}\left(b_{0 m j}\right)} .
$$

Furthermore, $\mu_{j}$ and $\sigma_{j}^{2}$ are estimated by the mean and variance of $\log b_{0 m j}$.

3. Plug-in estimator:

In equation (1), $P_{i j}$ and $B_{0 m j}$ are observable intensities. Therefore, the plug-in estimator is implemented by

(a) estimating $\alpha_{j}$ and $\beta_{j}$ through MLE based on the $p_{i j}$ values, and

(b) estimating $\mu_{j}$ and $\sigma_{j}^{2}$ through MLE based on the $b_{0 m j}$ values.

\subsection{Benchmarking}

Benchmarking data set Illumina platform has provided a benchmarking data set, the Illumina spike-in Dunning, Barbosa-Morais, Lynch, Tavaré, and Ritchie (2008). These spikein probes are targeting bacterial and viral genes absent from the mouse genome. These were added at specific concentrations on each sample. Therefore the change in expression level of a particular spike between samples is known a priori. The expression levels of the non-spikes should not change between samples.

There are twelve different concentrations of spike: 1000 picomolar $(\mathrm{pM}), 300,100,30,10$, $3,1,0.3,0.1,0.03,0.01$, and $0.00 \mathrm{pM}$. It was replicated four times. Therefore, there are 48 samples and each sample has regular and control bead-type level probes.

There are approximately about 48000 bead-type level probes for each sample and in addition the 33 spike-in bead-type level probes are added into it. For the control, there are 1616 beadtype level probes. These control experiments are the benchmarking data sets of Illumina and are used to compare low-level analysis methods such as in Affymetrix platform.

Performance studies We compare all convolution models: Irizarry et al. (2003a,b, 2006) and Bolstad et al. (2003): RMA (Exponential-Normal), Plancade et al. (2011, 2012): GammaNormal, Chen et al. (2011): Exponential-Gamma, Xie et al. (2009): Exponential-Normal adjusted for Illumina BeadArrays with MLE for the parameters, Bayesian approach and the method of moments, and the proposed models: exponential-lognormal and gamma-lognormal. We will call the methods above, respectively, as follows: ENr, GN, EG, ENm, ENm, ENn, ELNn, ELNm, ELNp, GLNn, GLNm, and GLNp. We use the MBCB package (Allen et al. (2009) and Xie et al. (2009)) to adjust the intensity values of these existing models ENr, ENm, ENmc and ENn. Except that, the GN uses the NormalGamma package (Plancade et al. (2011)).

Table 1 shows that the GLNn reproduces the Illumina concentration better than others. The ENr shows the closest performance toward the GLNn. Note that the computation of the Kullback-Leibler coefficient is implemented in each array $j$ based on the nominal concentrations $O$ in Table 1 and the observed intensities $P$ in Table 2, and the value in each 
Table 1: Reproducibility of each method toward the Illumina spike-in concentration

\begin{tabular}{lrr}
\hline Model & RMSE & K-L \\
\hline ENr & 1.346 & 51310 \\
ENn & 1.407 & 41010 \\
ENm & 1.483 & 23170 \\
ENmc & 1.483 & 23170 \\
EG & 1.470 & 20660 \\
GN & 1.521 & 58480 \\
ELNn & 1.411 & 41200 \\
ELNm & 1.489 & 21280 \\
ELNp & 1.423 & 37800 \\
GLNn & $\mathbf{1 . 3 2 3}$ & $\mathbf{4 3 3 3}$ \\
GLNm & 1.510 & 29630 \\
GLNp & 10.700 & -115400 \\
\hline
\end{tabular}

Table 2: Reproducibility of each method toward the Illumina spike-in based on the experiment data

\begin{tabular}{lrr}
\hline Model & RMSE & K-L \\
\hline ENr & 7.251 & 1141000 \\
ENn & 7.127 & 1062000 \\
ENm & 6.927 & 926500 \\
ENmc & 6.927 & 926200 \\
EG & 6.919 & 907900 \\
GN & 7.100 & 1183000 \\
ELNn & 7.124 & 1062000 \\
ELNm & 6.904 & 911600 \\
ELNp & 7.092 & 1035000 \\
GLNn & $\mathbf{6 . 8 2 5}$ & $\mathbf{7 9 3 4 0 0}$ \\
GLNm & 6.937 & 968400 \\
\hline
\end{tabular}

table is the median Kullback-Leibler coefficient based on $J=42$ arrays. The KullbackLeibler coefficient for two positive sequences $\left(X_{1 j}, \ldots, X_{I j}\right),\left(S_{1 j}, \ldots, S_{I j}\right)$ is computed as

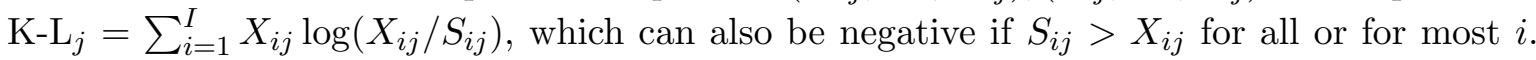
This is a sign that the $S$ are overestimating $X$, where $X$ could be $O$ (Table 1) or $P$ (Table 2). Therefore we exclude the GLNp model from further comparisons. The behavior of GLNp which is different from other models, also shown at the supplemental plots.

Table 2 shows how each method reproduces the data from the experiment. We see that GLNn can be considered to reproduce it better than others, based on the RMSE, and the KullbackLeibler coefficient. Tables 1 and 2 provide insight about how the performance comparison among the models would be conducted further.

In the first part, we compute the adopted Affycomp benchmarking criteria, based on the data after background correction and their log transformation. In the second part, in the simulation, the $\mathrm{MSE}_{b c}$ and the $L_{1}$ error will be computed based on the log transformation of the experiment and the nominal concentration data.

The log transformations that we use here, respectively, for the benchmarking and the FFPE data sets are as follows

$$
y=\log _{2}\left(x+\sqrt{x^{2}+1}\right) \quad \text { and } \quad y=\log _{2}\left(x+1+\sqrt{x^{2}+1}\right),
$$


Table 3: Median SD, IQR, and 99.9\% percentiles of log fold change for non spike-in between replicates for each model.

\begin{tabular}{lccc}
\hline Model & Median SD & IQR & $99.9 \%$ \\
\hline ENr & $\mathbf{0 . 0 2 7}$ & $\mathbf{0 . 0 6 2}$ & 0.415 \\
ENn & 0.043 & 0.089 & 0.441 \\
ENm & 0.069 & 0.139 & 0.486 \\
ENmc & 0.069 & 0.139 & 0.486 \\
EG & 0.065 & 0.134 & 0.477 \\
GN & 0.051 & 0.098 & 0.520 \\
ELNn & 0.045 & 0.093 & 0.442 \\
ELNm & 0.071 & 0.145 & 0.489 \\
ELNp & 0.049 & 0.100 & 0.449 \\
GLNn & 0.038 & 0.075 & $\mathbf{0 . 3 9 8}$ \\
GLNm & 0.076 & 0.080 & 0.507 \\
\hline
\end{tabular}

Table 4: The signal detect $R^{2}$ by regressing the nominal and observed value for each model for the Illumina spike-in.

\begin{tabular}{lcrrr}
\hline Model & $R^{2}$ & Low. $R^{2}$ & Med. $R^{2}$ & High. $R^{2}$ \\
\hline ENr & 0.959 & 0.618 & $\mathbf{0 . 6 9 8}$ & $\mathbf{0 . 5 5 9}$ \\
ENn & 0.958 & 0.622 & 0.695 & 0.557 \\
ENm & 0.957 & 0.635 & 0.695 & 0.558 \\
ENmc & 0.957 & 0.635 & 0.695 & 0.558 \\
EG & 0.957 & 0.633 & 0.695 & 0.558 \\
GN & 0.956 & $\mathbf{0 . 6 5 0}$ & 0.697 & 0.555 \\
ELNn & 0.958 & 0.624 & 0.695 & 0.557 \\
ELNm & 0.957 & 0.636 & 0.694 & 0.558 \\
ELNp & 0.958 & 0.627 & 0.695 & 0.557 \\
GLNn & $\mathbf{0 . 9 6 0}$ & 0.609 & 0.696 & 0.558 \\
GLNm & 0.956 & 0.637 & 0.694 & 0.558 \\
\hline
\end{tabular}

where $x$ is the nominal concentration $O$ or the observed intensity value $P$.

First part In Table 3 it is shown that the ENr method provides the smallest variation and IQR and the GLNn model provides the smallest 99.9\% percentiles of log fold change for the non spike-in between replicates. The largest variation, IQR, and 99.9\% percentiles, respectively, are observed for the GLNm, the ELNm, and the GN method.

In Table 4 it is shown that, in general, all methods perform similar to each other. The GLNn models have the highest signal detect $R^{2}$. The GN model has the highest $R^{2}$ at low concentration but has the lowest $R^{2}$ at high concentration. This means that the GN model works better at low concentration. On the other hand the ENr shows that it works better at medium and high concentrations, which is followed closely by GLNn model.

If we divide the concentrations into two categories, where high concentration means that the nominal concentration is at least $3 \mathrm{pM}$ and low concentration means that the nominal concentration is at most $1 \mathrm{pM}$, the GLNn model has the highest $R^{2}$ (the data is not shown here). It means, in general and at high concentrations, the GLNn offers a better fit than other models. As in Table 4, Table 5 shows that all models have similar performance, although the GLNn model has the highest $R^{2}$ of nominal concentration against observed log-fold-change. Table 6 provides the results from the computation of the AUC value. The table shows that all models have a better accuracy at medium concentrations than at low and high concentrations. The ENr performs very poor at the low concentrations, but the GLNm performs best. At high 
Table 5: The $R^{2}$ observed log-fold-change against nominal log-fold-changes for the spike-in genes.

\begin{tabular}{lcc}
\hline Model & Obs-intended-fc. $R^{2}$ & Obs-(low)-int-fc. $R^{2}$ \\
\hline ENr & 0.976 & 0.989 \\
ENn & 0.974 & 0.990 \\
ENm & 0.972 & 0.985 \\
ENmc & 0.972 & 0.985 \\
EG & 0.972 & 0.986 \\
GN & 0.970 & 0.987 \\
ELNn & 0.974 & 0.990 \\
ELNm & 0.972 & 0.985 \\
ELNp & 0.973 & 0.990 \\
GLNn & $\mathbf{0 . 9 7 8}$ & $\mathbf{0 . 9 9 1}$ \\
GLNm & 0.971 & 0.984 \\
\hline
\end{tabular}

Table 6: AUC value for each model.

\begin{tabular}{lccccc}
\hline Model & $\begin{array}{c}\text { Low } \\
\text { concentration AUC }\end{array}$ & $\begin{array}{c}\text { Medium } \\
\text { concentration AUC }\end{array}$ & $\begin{array}{c}\text { High } \\
\text { concentration AUC }\end{array}$ & $\begin{array}{c}\text { Average } \\
\text { AUC }\end{array}$ & All \\
\hline ENr & 0.450 & 0.987 & $\mathbf{0 . 7 8 5}$ & 0.585 & 0.886 \\
ENn & 0.518 & 0.987 & 0.764 & 0.631 & 0.899 \\
ENm & 0.573 & 0.987 & 0.741 & 0.667 & 0.911 \\
ENmc & 0.573 & 0.987 & 0.741 & 0.667 & 0.911 \\
EG & 0.567 & 0.987 & 0.746 & 0.664 & 0.910 \\
GN & 0.552 & 0.987 & 0.723 & 0.651 & 0.904 \\
ELNn & 0.524 & 0.987 & 0.763 & 0.635 & 0.900 \\
ELNm & 0.574 & 0.987 & 0.741 & 0.668 & 0.912 \\
ELNp & 0.534 & 0.987 & 0.761 & 0.642 & 0.902 \\
GLNn & 0.498 & $\mathbf{0 . 9 8 7}$ & 0.784 & 0.619 & 0.896 \\
GLNm & $\mathbf{0 . 5 7 9}$ & 0.987 & 0.730 & $\mathbf{0 . 6 7 1}$ & $\mathbf{0 . 9 1 3}$ \\
\hline
\end{tabular}

concentrations, the ENr performs the best and it is followed by the GLNn. But in general, the highest AUC is achieved by all the model with the MLE parameter estimation methods: the GLNm, ELNm, and ENm.

The computation, which is based on all arrays, provides the results where all models have the AUC greater than 0.9. According to Zhu, Zeng, and Wang (2010), the AUC between 0.9 and 1.0 is classified as excellent in measuring the accuracy. Therefore, based on Table 6 , we can identify that there are some models excellency accurate in predicting the gene expression.

Second part We do $N=100$ simulations to assess the performance of each model. The bias of the background correction is assessed by the $\mathrm{MSE}_{b c}$, and the bias of the parameterization is assessed by the $L_{1}$ error.

From the simulation results in Table 7 we can see that results for the EG model are not available, because the MBCB package did not work at the log transformation that we have chosen. The GN model performs best, by providing the smallest bias for the background correction and the parameters. A close performance is achieved by the ELN, particularly by the ELNn. The GLNn does not have an optimal performance on the $\mathrm{MSE}_{b c}$, but we still can consider its performance good, concerning that the bias of the parameters are similar to other proposed models and GN.

One of the proposed models, GLNm has the highest bias on the $\mathrm{MSE}_{b c}$ and the parameter $\alpha$. In our view this happens because we use an approximation in estimating the true intensity 
Table 7: The simulation results on the spike-in data set.

\begin{tabular}{lccccc}
\hline Model & MSE $_{b c}$ & \multicolumn{4}{c}{$L_{1}$ error } \\
\cline { 4 - 6 } & & $\alpha$ & $\beta$ & $\mu$ & $\sigma$ \\
\hline ENr & 0.045 & & 0.664 & 46.58 & 11.44 \\
ENn & 0.049 & & 0.625 & 41.61 & 2.806 \\
ENm & 0.038 & & 0.610 & 58.92 & 2.040 \\
ENmc & 0.036 & & 0.610 & 62.77 & 2.039 \\
GN & $\mathbf{0 . 0 3 0}$ & $\mathbf{0 . 0 0 0 3}$ & $\mathbf{0 . 0 0 7}$ & 0.013 & $\mathbf{0 . 0 1 5}$ \\
ELNn & 0.048 & & 0.009 & 0.0004 & 0.018 \\
ELNm & 0.039 & & 0.840 & 0.0004 & 0.018 \\
ELNp & 0.061 & & 0.472 & 0.0004 & 0.018 \\
GLNn & 0.216 & 0.052 & 0.055 & 0.0004 & 0.018 \\
GLNm & 84.37 & 38.86 & 0.851 & $\mathbf{0 . 0 0 0 3}$ & 0.017 \\
\hline
\end{tabular}

value. The EN models (ENr, ENm, ENn and ENmc) have considerably better performance at $\mathrm{MSE}_{b c}$, but are not good at the parametrization. The bias on the parametrization of the noise is higher than in other models.

\subsection{The public data sets}

Based on the results from Section 3.3, we compare the performance of these models on some public data sets. We would like to know how good these models are in real data samples. Here, we choose to use the formalin-fixed, paraffin-embedded (FFPE) data sets from Waldron, Ogino, Hoshida, Shima, Reed, Simpson, Baba, Nosho, Segata, Vargas, Cummings, Lakhani, Kirkner, Giovannucci, Quackenbush, Golub, Fuchs, Parmigiani, and Huttenhower (2012): the FFPE of tumors from colorectal cancer patients (GSE32651, 1003 samples), breast cancer metastases of the lymph node and autopsy tissues (GSE32490: GSE32489, 120 samples). Each sample has 24526 bead-type level probes.

The links for the data set are http://www.ncbi.nlm.nih.gov/geo/query/acc.cgi?acc= GSE32651 and http://www.ncbi.nlm.nih.gov/geo/query/acc.cgi?acc=GSE32490.

Currently the FFPE archival samples are widely available in million and it is a great source of information in medical studies about some diseases, for example cancer. This data type is suffering from the RNA degradation, which leads to poor performance in array-based studies. However, the Illumina's DASL assays could provide high-quality data from this degraded RNA samples.

Comparing the performance of these background correction models certainly would help researchers to choose the appropriate background correction for their data, particularly if their data is the FFPE type.

The background correction for the FFPE data set is implemented in three steps:

step 1 Do the quality control (QC) to the raw FFPE data. In this paper, we used the ffpe package in $\mathrm{R}$ (Waldron (2013)).

step 2 Do the data transformation $\log _{2}\left(P_{i j}+1+\sqrt{P_{i j}^{2}+1}\right.$ to the raw FFPE data after QC and estimate the background correction parameters based on it. The estimators of true intensity value and the background correction are based on the regular and negative control probe intensity data, respectively.

step 3 Compute the true intensity value (the adjusted intensity estimator) based on the BC parameters at step 2 . 
Table 8: Simulation results on the GSE32651 data set.

\begin{tabular}{lrrrrr}
\hline Model & MSE $_{b c}$ & \multicolumn{4}{c}{$L_{1}$ error } \\
\cline { 3 - 6 } & & $\alpha$ & $\mu$ & $\sigma$ \\
\hline ENr & 0.058 & & 0.657 & 297.67 & 22.61 \\
ENn & 0.281 & & 0.572 & 1.984 & 2.627 \\
ENm & 0.086 & & 0.591 & 28.05 & 1.715 \\
ENmc & $\mathbf{0 . 0 3 6}$ & & 0.610 & 62.77 & 2.039 \\
ELNn & 0.275 & & $\mathbf{0 . 0 2 5}$ & 0.001 & 0.018 \\
ELNm & 0.059 & & 0.826 & $\mathbf{0 . 0 0 0 4}$ & 0.018 \\
ELNp & 0.672 & & 0.487 & 0.001 & 0.018 \\
GLNn & 0.838 & $\mathbf{0 . 3 4 0}$ & 0.527 & 0.001 & 0.018 \\
GLNm & 84.15 & 71.87 & 0.887 & 0.0005 & $\mathbf{0 . 0 1 8}$ \\
\hline
\end{tabular}

Table 9: Simulation results on the GSE32489 data set.

\begin{tabular}{lrrrrr}
\hline Model & MSE $_{b c}$ & \multicolumn{4}{c}{$L_{1}$ error } \\
\cline { 3 - 6 } & & $\alpha$ & $\mu$ & $\sigma$ \\
\hline ENr & $\mathbf{0 . 0 9 3}$ & & 0.665 & 67.17 & 9.511 \\
ENn & 0.863 & & 0.509 & 0.936 & 2.039 \\
ENm & 0.182 & & 0.558 & 14.20 & 1.712 \\
ENmc & 0.184 & & 0.556 & 14.18 & 1.049 \\
ELNn & 1.055 & & 0.857 & 0.002 & 0.018 \\
ELNm & 0.116 & & 0.781 & 0.001 & 0.018 \\
ELNp & 1.247 & & 0.461 & 0.002 & 0.018 \\
GLNn & 1.348 & $\mathbf{0 . 3 3 2}$ & $\mathbf{0 . 4 9 7}$ & 0.002 & $\mathbf{0 . 0 1 8}$ \\
GLNm & 164.98 & 22.24 & 0.805 & $\mathbf{0 . 0 0 0 4}$ & $\mathbf{0 . 0 1 8}$ \\
\hline
\end{tabular}

The results of our computation are in Tables 8 and 9. From these tables we can see that there are no EG and GN models. Neither of these models can work on these data sets. For some samples in the data set, both models fail to compute the parameters which has the consequence that the true intensity value cannot be provided.

We decided to remove the EG and GN models from further comparisons in both FFPE data sets. Here we provide the results of the rest of the models only.

Tables 8 and 9 consistently show that the bias of the parameters of noise in the EN models are higher than the proposed models. For the parameter $\beta$, the ELNn has the smallest bias and it is followed by the ELNp and the GLNn. With regard to the bias of the background correction, the EN models show the smallest bias in both of FFPE data sets.

\section{Conclusions and indication of future work}

We have studied additive models of background correction for BeadArrays and proposed some new models where the true intensity is assumed to have exponential or gamma distribution and the noise is lognormally distributed. We have derived the estimator of the true intensity value of the proposed models.

Further, we compared the performance of all models, based on the benchmarking and public data sets. In the benchmarking data set we adopted the criteria from the Affycomp (Cope et al. 2004) and for the simulation study we used the criteria which have been used in Xie et al. (2009), Chen et al. (2011) and Plancade et al. (2011, 2012). For the public data sets, we only used the criteria for the simulation study.

We have seen in Sections 3.3 and 3.3 that EN, EG, GN and GLN perform rather similar. However, the GLNn model has provided the highest reproducibility in comparison to other 
models. From the Affycomp criteria we can provide the following points:

1. The ENr and GLNn provide the lowest variation between replicates and all models using the MLE estimation method have a higher variation than others.

2. The GLNn model has the highest signal detect $R^{2}$ in general and in high concentration. This means the GLNn model is the best fitted for the gene expression.

3. The GLNn model, based on the MvA plot, produces the least number of genes which should not be expressed but are nevertheless expressed. On the other hand, the GN model provides the largest number of such genes.

4. All models with the MLE estimation method have a higher average AUC value, which means that they provide a better accuracy in predicting the gene expression.

5. The ENr and GLNn have the lowest IQR of log fold-change between replicates.

6. Points 1 and 2 show that the GLNn and ENr are more accurate and precise in modelling the gene expression and points 3 and 5 show that the specificity and sensitivity of the GLNn and ENr model are better than others.

In the simulation study, the best performance in estimating the signal by measuring its background correction and parametrization errors is achieved by the GN model. It is followed by our proposed ELN models. It has been shown that the GLNn does not perform optimally at the $\mathrm{MSE}_{b c}$ criterion, but for the parametrization this model still can be considered good.

In the FFPE public data set, the GN and EG models cannot be implemented. This is in strong contrast with the fact that in the simulation study of benchmarking data set, the GN model has the best performance.

The EN models show the highest bias in the parametrization in both public data sets and the lowest bias in the background correction. Our proposed models, except the GLNm, show the lowest bias in the parametrization in both data sets and a moderate bias in the background correction.

Based on the results from the benchmarking data and the public data sets, we would suggest researchers the following:

1. if the GN model works properly at the data set at hand (i.e. the estimated signals in all arrays can be computed by this model and the simulation criteria for this data at this model are low) then use the GN model to correct the background.

2. if the GN model fails, then use our proposed models, particularly the GLNn model. The reason for not choosing the ELN models is that the value of the parameter $\alpha$ from the benchmarking data set is less than 1, around 0.2. Therefore, the gamma model is more appropriate to model the true intensity distribution than the exponential one. We believe that the right approximative computation of the GLN models will lead to a better performance than the current approximation.

The ELN models perform better than the original EN models, due to the fact that not only the regular probes, but also the control probes are skew-distributed (Chen et al. 2011). Therefore, these models could be the second choice after the GLN, when the GN model does not work.

3. With regard to the computation time, at the benchmarking data set the EN models are working faster than the others. They are followed by the ELNp, ELNn, and EG. The GLNn and the ENmc are the third fastest, then come the GN and the ELNm, which are followed by the GLNm as the slowest one. 
One of the purposes of using microarray technology is finding the genes which are expressed differentially due to some disease or condition. Therefore, it is important to investigate the effect of bias of the background correction and the parametrization toward the differentially expressed genes. This will be our future work.

\section{Acknowledgements}

This paper is part of the author's Ph.D dissertation written under the direction of Prof. István Berkes. We would like to thank Herwig Friedl, Ernst Stadlober, Levi Waldron, and an anonymous referee for their comments leading to a substantial improvement of the paper. We thank Florian Endel for discussion and suggestion how to make the R programming more efficient. We thank Yevgeniy Grigoryev and Ken Laing for their generous permission to use their pictures in the Supplementary material at http://rfajriyah.staff.uii.ac. id/category/supplementary/. Financial support from the Austrian Science Fund (FWF), Project P24302-N18 is gratefully acknowledged.

\section{References}

Allen JD, Chen M, Xie Y (2009). "Model-Based Background Correction (MBCB): R Methods and GUI for Illumina Bead-array Data." Journal of Cancer Science and Therapy, 1(1), 2527.

Baek J, Son YS, MacLachlan GJ (2007). "Segmentation and Intensity Estimation of Microarray Images Using a Gamma-t Mixture Mode." Bioinformatics, 23(4), 458-465.

Bolstad BM (2004). Low Level Analysis of High-Density Oligonucleotide Array Data: Background, Normalization and Summarization. Ph.D. thesis, University of California, California, Berkeley.

Bolstad BM, Irizarry RA, Astrand M, Speed TP (2003). "A Comparison of Normalization Methods for High Density Oligonucleotide Array Data Based on Bias and Variance." Bioinformatics, 19(2), 185-193.

Chen M, Xie Y, Story MD (2011). "An Exponential-Gamma Convolution Model for Background Correction of Illumina BeadArray Data." Communication in Statistics: theory and methods, 40(17), 3055-3069.

Cope LM, Irizarry RA, Jaffee HA, Wu Z, Speed TP (2004). "A Benchmark for Affymetrix GeneChip Expression Measures." Bioinformatics, 20, 323-331.

Ding LH, Xie Y, Park S, Xiao G, Story MD (2008). "Enhanced Identification and Biological Validation of Differential Gene Expression via Illumina Whole-Genome Expression Arrays through the Use of the Model-Based Background Correction Methodology." Nucleic Acids Research, 36(10: e58).

Dunning MJ, Barbosa-Morais NL, Lynch AG, Tavaré S, Ritchie ME (2008). "Statistical Issues in the Analysis of Illumina Data." BMC Bioinformatics, 9(85).

Forcheh AC, Verbeke G, Kasim A, Lin D, Shkedy Z, Talloen W, Gohlmann HW, Clement L (2012). "Gene Filtering in the Analysis of Illumina Microarrays Experiments." Statistical Applications in Genetics and Molecular Biology, 11(2).

Hochreiter S, Djork-Arné, Obermayer K (2006). "A New Summarization Method for Affymetrix Probe Level Data." Bioinformatics, 22(8), 943-949.

Huber W, Irizarry RA, Gentleman R (2005a). Bioinformatics and Computational Biology Solutions Using $R$ and Bioconductor, chapter Preprocessing Overview. Springer. 
Huber W, von Heydebreck A, Vingron M (2004). "Error Models for microarray Intensities." Technical Report Paper 6, Bioconductor Project Working Papers.

Huber W, von Heydebreck A, Vingron M (2005b). "An Introduction to Low-Level Analysis Methods of DNA Microarray Data." Technical Report Paper 9, Bioconductor Project Working Papers.

Irizarry RA, Bolstad BM, Collin F, Cope LM, Hobbs B, Speed TP (2003a). "Summaries of Afymetrix GeneChip Probe Level Data." Nucleic Acids Research, 31(4).

Irizarry RA, Hobbs B, Collin F, Beazer-Barclay YD, Antonellis KJ, Scherf U, Speed TP (2003b). "Exploration, Normalization and Summaries of High Density Oligonucleotide Array Probe Level Data." Biostatistics, 4(2), 249-264.

Irizarry RA, Wu Z, Jaffee HA (2006). "Comparison of Affymetrix geneChip Expression Measures." Bioinformatics, 22(7), 789-794.

Li C, Wong WH (2001). "Model-Based Analysis of Oligonucleotide Arrays: Expression Index Computation and Outlier Detection." Proceeding national Academy of Sciences, 98(1), $31-36$.

Plancade S, Rozenholc Y, Lund E (2011). "Improving Background Correction for Illumina BeadArrays: the Normal-Gamma Model."

Plancade S, Rozenholc Y, Lund E (2012). "Generalization of the Normal-Exponential Model: Exploration of a More Accurate Parameterisation for the Signal Distribution on Illumina BeadArrays." BMC Bioinformatics, 13(329).

Shi W, Oshlack A, Smyth GK (2010). "Optimizing the Noise versus Bias Trade-off for Illumina Whole Enome Expression Beadchips." Nucleic Acids Research, 38(22: e204).

Silver JD, Ritchie ME, Smyth GK (2009). "Microarray Background Correction: Maximum Likelihood Estimation for the Normal-Exponential Convolution Model." Biostatistics, 10, $352-363$.

Triche TJ, Weisenberger DJ, Berg DVD, Laird PW, Siegmund KD (2013). "Low-level Processing of Illumina Infinium DNA Methylation BeadArrays." Nucleic Acids Research, pp. $1-11$.

Waldron L (2013). ffpe: Quality Assessment and Control for FFPR Microarray Expression Data, r package version 1.4.0 edition.

Waldron L, Ogino S, Hoshida Y, Shima K, Reed AEM, Simpson PT, Baba Y, Nosho K, Segata N, Vargas AC, Cummings M, Lakhani SR, Kirkner GJ, Giovannucci E, Quackenbush J, Golub TR, Fuchs CS, Parmigiani G, Huttenhower C (2012). "Expression Profiling of Archival Tumors for Long-term Health Studies." Clinical Cancer Research.

Wu Z, Irizarry RA, Gentleman R, Martinez-Murillo F, Spencer F (2004). "A Model-Based Background Adjustment for Oligonucleotide Expression Arrays." Journal of the American Statistical Association, 99(468), 909 - 917.

Xie Y, Wang X, Story MD (2009). "Statistical Methods of Background Correction for Illumina BeadArray Data." Bioinformatics, 25(6), 751-757.

Zhang J (2013). "Reducing the Bias of the Maximum Likelihood Estimator of the Shape Parameter for the Gamma Distribution." Computational Statistics, 28, 1715 - 1724.

Zhu W, Zeng N, Wang N (2010). "Sensitivity, Specificity, Accuracy, Associated Confidence Interval and ROC Analysis with Practical SAS Implementations." Http://www.nesug.org/Proceedings/nesug10/hl/hl07.pdf. 


\section{Affiliation:}

Rohmatul Fajriyah

Institute of Statistics

Graz University of Technology

8010 Graz, Austria

E-mail: fajriyah@student.tugraz.at

Department of Statistics

Universitas Islam Indonesia

Jl. Kaliurang Km. 14.4

55584 Jogjakarta, Indonesia

E-mail: rfajriyah@fmipa.uii.ac.id

URL: http://rfajriyah.staff.uii.ac.id/

\section{Austrian Journal of Statistics}

published by the Austrian Society of Statistics

Volume 45

March 2016 http://www .ajs.or.at/

http://www.osg.or.at/

Submitted: 2014-11-13

Accepted: 2014-11-13 